\title{
Suppressed protein expression of the death-associated protein kinase enhances 5-fluorouracil-sensitivity but not etoposide- sensitivity in human endometrial adenocarcinoma cells
}

\author{
TETSUJI TANAKA ${ }^{1}$, TAO BAI $^{1}$ and KAZUNORI YUKAWA ${ }^{2}$ \\ ${ }^{1}$ Department of Obstetrics and Gynecology, Wakayama Medical University, 811-1 Kimi-idera, Wakayama 641-0012; \\ ${ }^{2}$ Department of Physiology, Faculty of Pharmacy, Meijo University, 150 Yagotoyama, Tempaku-ku, Nagoya 468-8503, Japan
}

Received June 8, 2010; Accepted July 30, 2010

DOI: 10.3892/or_00000999

\begin{abstract}
Targeted knockdown of the death-associated protein kinase (DAPK) expression in the endometrial adenocarcinoma HHUA cells reportedly induces cell death by enhancing the tumor necrosis factor-related apoptosisinducing ligand (TRAIL)-mediated apoptosis in an autocrine/ paracrine manner. This suggests that endogenous DAPK is a potential candidate for a molecularly targeted anticancer therapy for patients with endometrial adenocarcinoma. To investigate the role of endogenous DAPK in anticancer drug sensitivity, we examined effects on cellular anticancer drug sensitivities of transfections with 5 different specific DAPK small-interfering RNAs (siRNAs) into HHUA cells. DAPK siRNA transfections strongly enhanced 5-fluorouracil (5FU)sensitivity, but not etoposide-sensitivity, of HHUA cells compared with control siRNA-transfected cells. These results indicate that etoposide-stimulated cell death signals may share or include TRAIL-mediated apoptotic signals, and that 5FU-stimulated cell death signals may be independent from TRAIL-mediated apoptotic signals induced by DAPK siRNA transfections. Moreover, 5FU-combined chemotherapy with DAPK siRNA transfection may show stronger anticancer effects on patients with endometrial adenocarcinoma than does chemotherapy alone.
\end{abstract}

\section{Introduction}

The death-associated protein kinase (DAPK) cDNA was isolated as a positive mediator of apoptosis triggered by IFN- $\gamma$ from human cervical carcinoma cells (1). Investigations have revealed that DAPK functions as a $\mathrm{Ca}^{2+} /$ calmodulindependent serine/threonine kinase to regulate cell death or cell survival (1-14). However, DAPK's physiological functions

Correspondence to: Dr Tetsuji Tanaka, Department of Obstetrics and Gynecology, Wakayama Medical University, 811-1 Kimi-idera, Wakayama 641-0012, Japan

E-mail: obgywmu@wakayama-med.ac.jp

Key words: death-associated protein kinase, 5-fluorouracil, drug sensitivity, etoposide, cisplatin have not been fully clarified. Loss of DAPK expression has been implicated in tumorigenesis and metastasis $(3,4,13)$, thereby suggesting a crucial role for DAPK in the apoptotic process under pathological conditions. On the other hand, inhibition of DAPK expression in HeLa cells, 3T3 fibroblasts and primary human vascular smooth muscle cells with an antisense DAPK was found to increase apoptosis $(6,12)$.

In a human cervical squamous cell carcinoma cell line ME180, DAPK protein expression is constitutively suppressed but can be strongly induced by treatment with a demethylation agent, 5-aza-2'-deoxycitidine (5-aza-CdR), and a histone deacetylation inhibitor, trichostatin (15). However, in ME180derived cisplatin (CDDP)-resistant cell lines, DAPK protein expression can not be induced by treatment with 5-aza$\mathrm{CdR}$ and trichostatin. Although DAPK mRNA is expressed in the CDDP-resistant cells as in ME180 parent cells, DAPK mRNA translation is strongly suppressed in CDDP-resistant cells (15). These facts suggest that strong suppression of DAPK protein induction is involved in acquisition of CDDP resistance, and that DAPK protein regulates anticancer drug sensitivity and/or acquired anticancer drug resistance in cancer cells.

We recently reported that targeted knockdown of DAPK protein expression in the HHUA cell line (16), a highly differentiated endometrial adenocarcinoma cell line, induced cell death by enhancing tumor necrosis factor-related apoptosis-inducing ligand (TRAIL)-mediated apoptosis in an autocrine/paracrine manner (17). These findings suggest that endogenous DAPK is a candidate for molecularly targeted anticancer therapy for patients with endometrial adenocarcinoma, and that targeted knockdown of DAPK protein expression may enhance anticancer drug sensitivity in cancer cells. Therefore, we utilized specific DAPK small-interfering RNAs (siRNAs) to knock down endogenous DAPK protein expression in HHUA cells to directly investigate the role of endogenous DAPK in anticancer drug sensitivity.

\section{Materials and methods}

Cell line and culture. The HHUA cell line (16) was obtained from Riken Cell Bank (Tsukuba, Japan). Cells were cultured in OPTI-MEM (Invitrogen Corp., Carlsbad, CA) supplemented with $5 \%$ fetal bovine serum (FBS) (Equitech Bio Inc., Ingram, 
Table I. DAPK siRNA sequences.

\begin{tabular}{ll}
\hline Number of siRNA & \multicolumn{1}{c}{ siRNA sequence } \\
\hline DAPK siRNA 001 & 5'-CAACAUCAUGCAAAGUGAAACAGUU-AG-3' \\
DAPK siRNA 002 & ''-AU-GUUGUAGUACGUUUCACUUUGUCAA-5' \\
& 5'-AGCCAAGAAUUAAGCUCAAGCUGUU-AG-3' \\
5APK siRNA 003 & '-AU-UCGGUUCUUAAUUCGAGUUCGACAA-5' \\
& 5'-CUGUCAACUACGAAUUUGAGGAUGA-AG-3' \\
DAPK siRNA 004 & 3'-AU-GACAGUUGAUGCUUAAACUCCUACU-5' \\
& 5'-GAUCAAGCCUAAAGAUACACAACAG-AG-3' \\
DAPK siRNA 005 & 3'-AU-CUAGUUCGGAUUUCUAUGUGUUGUC-5' \\
& 5'-GGAUCAAGCCUAAAGAUACACAACA-AG-3' \\
\hline
\end{tabular}

TX), penicillin $(100 \mathrm{U} / \mathrm{ml})$, streptomycin $(100 \mathrm{U} / \mathrm{ml})$ and Fungizone $\left(0.25 \mu \mathrm{g} / \mathrm{ml}\right.$; Invitrogen Corp.) in $5 \% \mathrm{CO}_{2}$ and $95 \%$ air at $37^{\circ} \mathrm{C}$.

Transfection of DAPK siRNAs. Five DAPK siRNA duplexes were designed and synthesized by iGENE Therapeutics Inc. (Tsukuba, Japan). The 5 siRNA sequences are shown in Table I. A negative control siRNA was purchased from Ambion Inc. (Austin, TX). Lipofectamine 2000 (Invitrogen Corp.) was used as the transfection reagent according to the manufacturer's instructions. For experiments, cells were seeded in 6 -well plates $\left(2.5 \times 10^{5}\right.$ cells/well $)$ or $10-\mathrm{cm}$ dishes $\left(2 \times 10^{6}\right.$ cells/dish), cultured for $24 \mathrm{~h}$ and then transfected with the DAPK siRNAs or control siRNA at a final concentration of 25 or $50 \mathrm{nM}$. Subsequently, the cells were cultured for 24-48 $\mathrm{h}$ for mRNA analyses and 48-72 $\mathrm{h}$ for protein assays before being harvested as indicated.

Anticancer drug-sensitivity assays. All anticancer drugs were kind gifts from pharmaceutical companies. 5-fluorouracil (5FU) was provided by Kirin-Kyowa-Hakko Co., Ltd. (Tokyo, Japan). Cisplatin (CDDP) was provided by NihonKayaku Co., Ltd. (Tokyo, Japan). Etoposide (VP16) was provided by Bristol-Myers Squibb Japan Co., Ltd. (Tokyo, Japan). The effects of anticancer drugs on cell growth were assayed as follows. Cells in the log phase were detached with $0.25 \%$ trypsin/ $1 \mathrm{mM}$ EDTA (Gibco-BRL) and cultured overnight in 96 -well plates $\left(5 \times 10^{3}\right.$ cells/well). On day 2 , various concentrations of anticancer drugs were added to the cells. On day 4 , the numbers of viable cells were evaluated using a cell proliferation assay kit (Dojin, Tokyo, Japan) and expressed as the percentages of viable cells (\%) relative to the mean number of viable untreated cells. All experiments were performed in triplicate. Data are expressed as the mean $\pm S D$, and comparative data $(n=6)$ were analyzed by ANOVA.

Western blotting. For Western blot analysis, the cells were collected at 48-72 $\mathrm{h}$ after transfection with the DAPK siRNAs or control siRNA and lysed in phosphate-buffered
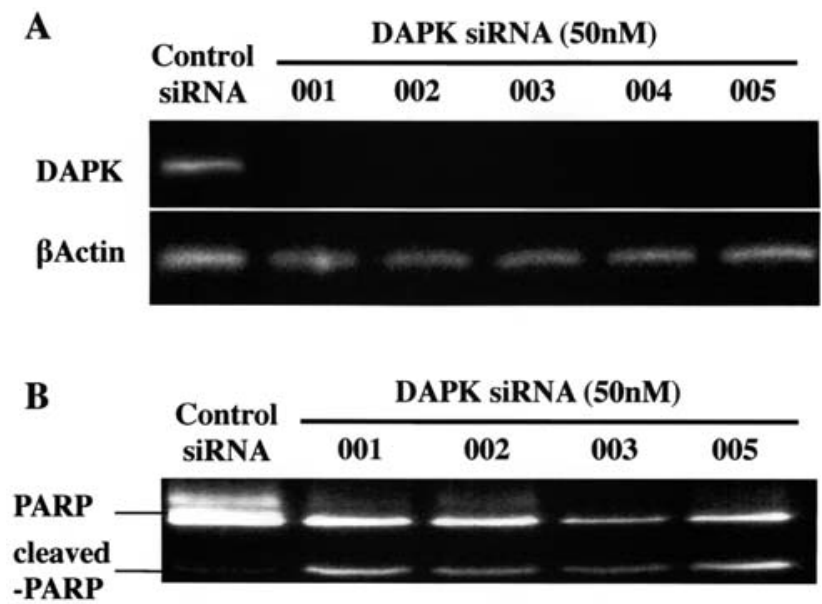

Figure 1. Western blot analysis of DAPK siRNA-transfected HHUA cells. (A) DAPK protein expression was strongly suppressed by transfection by each of 5 DAPK siRNAs. (B) Cleavage of PARP in DAPK siRNAtransfected cells

saline containing $1 \%$ NP-40, $0.1 \%$ sodium dodecyl sulfate, Complete protease inhibitor cocktail (Roche Diagnostics, Corp., Indianapolis, IN) and $1 \mathrm{mM}$ phenylmethyl sulfonyl fluoride. Protein concentrations of the cell lysates were quantified by Coomassie Plus Protein assays (Pierce Biotechnology, Inc., Rockford, IL). Equal amounts of the total proteins were separated by SDS-PAGE using a $7.5 \%$ gel and then transferred to a polyvinylidene fluoride membrane (Atto, Corp., Tokyo, Japan). After sequential incubations with primary and secondary antibodies, the immunocomplexes on the membranes were detected using enhanced chemiluminescence (ECL) or ECL plus kits (Amersham Pharmacia Biotech, Uppsala, Sweden). The antibodies used were purchased from the following sources: mouse monoclonal anti-DAPK antibody (Sigma, St. Louis, MO); rabbit polyclonal anti-poly (ADP-ribose) polymerase (PARP) and anti-cleaved PARP antibodies (Cell Signaling Technology, Beverly, MA). The membranes were stripped and reprobed with an anti- $\beta$-actin antibody (Sigma). 


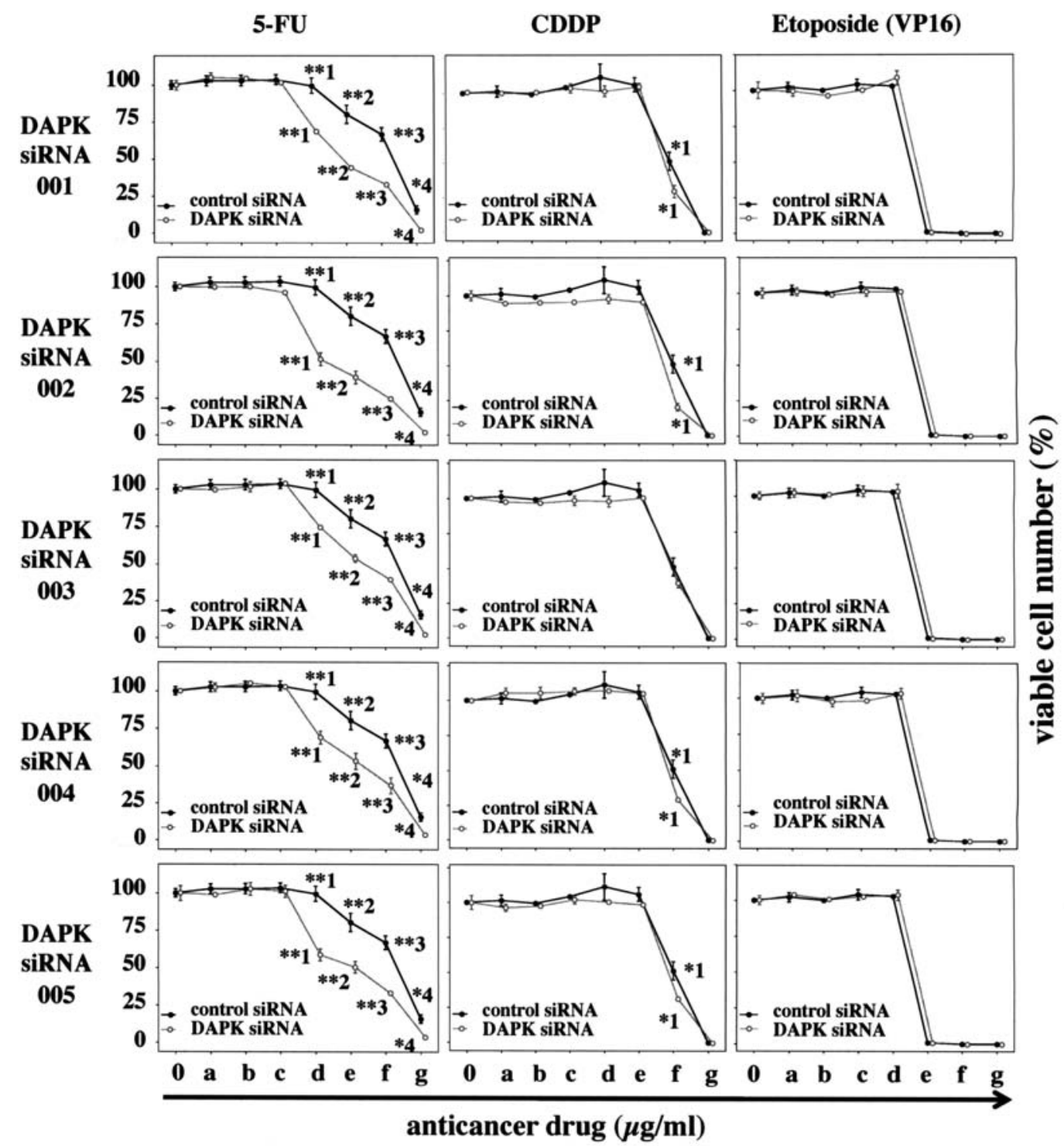

Figure 2. Anticancer drug sensitivity in the DAPK siRNA-transfected HHUA cells. Final concentrations $(\mu \mathrm{g} / \mathrm{ml})$ of each anticancer drug $(0$ and a-g at the bottom of figures), were as follows: $5 \mathrm{FU}, 0,0.128,0.64,3.2,16,80,400$ and $2000 \mu \mathrm{g} / \mathrm{ml}$; CDDP, 0, 0.0064, 0.032, 0.16, 0.8, 4, 20 and 100 $\mu \mathrm{g} / \mathrm{ml}$; VP16, $0,0.05120 .256,1.28,6.4,32,160$ and $800 \mu \mathrm{g} / \mathrm{ml} .{ }^{*} 1-{ }^{*} 3, \mathrm{P}<0.05 ;{ }^{* * *} 1{ }^{* *} 4, \mathrm{P}<0.01,{ }^{* * * *} 1{ }^{* * * *} 5, \mathrm{P}<0.001$.

RNase protection assay (RPA). Total RNA was isolated from the cultured cells using an RNeasy Mini Kit (Qiagen Inc., Valencia, CA) for RNase protection assays. A multiprobe RPA was performed using a RiboQuant ${ }^{\mathrm{TM}}$ hcc-2 Multiprobe Template Set and an RPA Kit (BD PharMingen) according to the manufacturer's protocol. Biotin-conjugated probes were prepared using a Non-Rad In Vitro Transcription Kit (BD PharMingen). The probes were combined with $10 \mu \mathrm{g}$ of total RNA isolated from DAPK siRNA-transfected or control siRNA-transfected HHUA cells. After denaturation at $95^{\circ} \mathrm{C}$ for $3 \mathrm{~min}$, the mixtures were allowed to hybridize at $56^{\circ} \mathrm{C}$ for 12-16 h before RNase digestion. RNA hybrids were separated in $4.75 \%$ acrylamide/ $8 \mathrm{M}$ urea denaturing gels, and the protected fragments were transferred to positively charged nylon membranes. The fragments on the membranes were detected using the RPA Kit and the protected fragments were quantified with a Luminocapture apparatus and lane analyzer software (ATTO Corp.). The relative expression of each mRNA species was calculated after normalization by the expression of the housekeeping gene L32.
Statistical analysis. The data were expressed as means \pm SD. Comparisons between experimental groups were performed by analysis of variance (ANOVA). If the ANOVA was significant, post-hoc comparisons were conducted using Scheffe's test. Statistical significance was set at $\mathrm{P}<0.05$.

\section{Results}

All 5 DAPK siRNA-transfected HHUA cells showed strong suppression of DAPK protein expression (Fig. 1A). Concurrently, specific downregulation of endogenous DAPK expression by DAPK siRNA transfection induced cleavage of PARP, an apoptosis marker, in the cells (Fig. 1B). No non-specific inhibitory effects of DAPK siRNA were detected on $B$-actin protein expression, used as an internal control (Fig. 1A).

Next, anticancer drug-sensitivity tests of the DAPK siRNA-transfected HHUA cells were performed using 3 drugs, 5FU, CDDP and VP16. The anticancer drug-sensitivity curves were compared with those of the control siRNA-transfected 


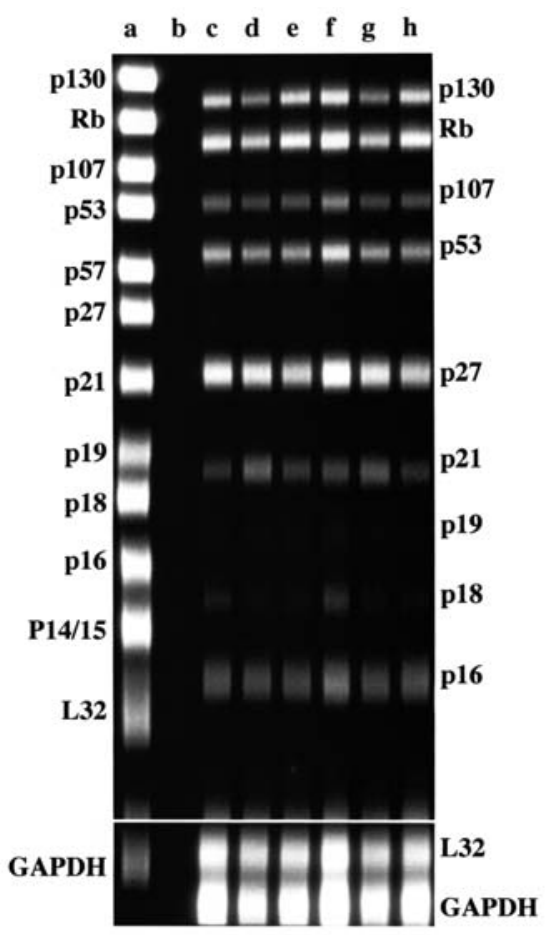

Figure 3. RNase protection assays for effects of the DAPK siRNA transfections on mRNA expressions of cell cycle-regulating molecules in HHUA cells. DAPK siRNA-transfected cells show no difference in mRNA expression for cell cycle-regulating molecules compared with control siRNA-transfected cells. The panel shows a representative set of electrophoresis data for the DAPK- and control-siRNA-transfected cells Lane a, unprotected probe; lane b, yeast tRNA; lane c, $50 \mathrm{nM}$ control siRNA-transfected cells; lane d, 50 nM DAPK siRNA 002-transfected cells; lane e, $50 \mathrm{nM}$ DAPK siRNA 004-transfected cells; lane f, $25 \mathrm{nM}$ control siRNA-transfected cells; lane g, 25 nM DAPK siRNA 002-transfected cells; and lane h, 25 nM DAPK siRNA 004-transfected cells.

HHUA cells. As shown in Fig. 2, all 5 different DAPK siRNAtransfected cells exhibited much higher 5FU-sensitivities than the control siRNA-transfected cells. However, VP16sensitivities in any DAPK siRNA-transfected cells could not be enhanced at all. As for CDDP-sensitivities of the DAPK siRNA-transfected cells, transfections with 4 of 5 DAPK siRNAs slightly enhanced CDDP-sensitivity while DAPK siRNA 003-transfected cells did not show any enhancement of CDDP-sensitivity compared with control siRNA-transfected cells.

$5 \mathrm{FU}$ is a well known time-dependent anticancer drug. Therefore, to investigate the mechanisms of enhanced 5FU-sensitivity by DAPK siRNA transfections, RPA was done to examine mRNA expressions of cell cycle-regulating molecules in the DAPK siRNA-transfected cells. As shown in Fig. 3, DAPK siRNA transfections did not affect 7 mRNA expressions (p130, Rb, p107, p53, p27, p21, p16) in DAPK siRNA-transfected cells, although p18 mRNA expression was apparently suppressed in the cells.

\section{Discussion}

This is the first report to directly show that suppressed DAPK protein expression can significantly enhance 5FU-sensitivity. Our recent research showed that DAPK siRNA transfections induce cell deaths in human endometrial adenocarcinoma cells, uterine carcinosarcoma cells and uterine leiomyosarcoma cells (18). In a human endometrial adenocarcinoma cell line HHUA, DAPK siRNA transfection induces TRAILmediated apoptosis in autocrine/paracrine manner (17). In the present study, we have shown that DAPK siRNA transfections strongly enhance 5FU-sensitivity, but not VP16sesitivity, in HHUA cells. These results indicate that DAPK can be a convincing target for anticancer therapy, and that 5FU-combined chemotherapy with DAPK siRNA transfection may have a stronger anticancer effect on patients with endometrial adenocarcinoma than the chemotherapy alone.

Our recent study showed that DAPK siRNA transfections into HHUA cells induce TRAIL-mediated apoptosis in cells (17). Since DAPK siRNA transfections did not affect VP16sensitivity in the cells at all, the VP16-stimulated cell death signals may share or include TRAIL-mediated apoptotic signals. On the other hand, findings that DAPK siRNA transfections strongly enhance 5FU-sensitivity suggest that 5FUstimulated cell death signals may be independent from the TRAIL-mediated apoptotic signals induced by DAPK siRNA transfections.

As shown in Fig. 2, transfections with 4 out of 5 DAPK siRNA slightly enhanced CDDP-sensitivity of HHUA cells. In a previous study, strong suppression of DAPK protein induction was shown to be involved in acquired CDDPresistance in ME180 cells (15). Contrary to this report, the present study has showed that CDDP-sensitivity was slightly enhanced in the DAPK siRNA-transfected cells. Taken together, these results present a disagreement between DAPK protein expression and CDDP-sensitivity between in HHUA cells and in ME180 cells. The disagreement may be caused by the cell lineage of the examined cancer cells, because DAPK is known to function as a positive or negative mediator in different apoptotic signals. Therefore, when DAPK is used for a molecular target in anticancer therapy, it should be first examined whether DAPK molecules play antiapoptotic roles in the target cells.

$5 \mathrm{FU}$ is a major anticancer drug that is clinically used for patients with various types of cancers. Because 5FU is a well-known time-dependent anticancer drug, we have examined RNA expressions of cell cycle-regulating molecules in the DAPK siRNA-transfected cells by RPA. As shown in Fig. 3, only p18 mRNA was significantly suppressed by DAPK knockdown. Although p18, a member of cyclindependent kinase inhibitor, is reported to be a tumor suppressor gene (19-21), further investigations are needed to clarify whether suppressed p18 expression can affect anticancer drug sensitivity of the cells.

\section{References}

1. Deiss LP, Feinstein E, Berissi H, Cohen O and Kimchi A: Identification of a novel serine/threonine kinase and a novel $15-\mathrm{kD}$ protein as potential mediators of the gamma interferoninduced cell death. Genes Dev 9: 15-30, 1995.

2. Cohen O, Feinstein E and Kimchi A: DAP kinase is a $\mathrm{Ca}^{2+}$ calmodulin-dependent, cytoskeleton-associated protein kinase, with cell death-inducing functions that depend on its catalytic activity. EMBO J 16: 998-1008, 1997.

3. Kissil JL, Feinstein E, Cohen O, Jones PA, Tsai YC, Knowles MA, Eydmann ME and Kimchi A: DAP kinase loss of expression in various carcinoma and B-cell lymphoma cell lines: possible implications for role as tumor suppressor gene. Oncogene 15: 403-407, 1997. 
4. Inbal B, Cohen O, Polak-Charcon S, Kopolovic J, Vadai E, Eisenbach L and Kimchi A: DAP kinase links the control of apoptosis to metastasis. Nature 390: 180-184, 1997.

5. Cohen O, Inbal B, Kissil JL, Raveh T, Berissi H, SpivakKroizaman T, Feinstein E and Kimchi A: DAP kinase participates in TNF-alpha- and Fas-induced apoptosis and its function requires the death domain. J Cell Biol 146: 141-148, 1999.

6. Jang $\mathrm{CW}$, Chen $\mathrm{CH}$, Chen CC, Chen JY, Su YH and Chen RH: TGFB induces apoptosis through Smad-mediated expression of DAP kinase. Nat Cell Biol 4: 51-58, 2002.

7. Raveh T, Droguett G, Horwitz MS, De Pinho RA and Kimchi A: DAP kinase activates a p19ARF/p53-mediated apoptotic checkpoint to suppress oncogenic transformation. Nat Cell Biol 3: $1-7,2001$.

8. Pelled D, Raveh T, Riebeling C, Fridkin M, Berissi H, Futerman AH and Kimchi A: Death-associated protein (DAP) kinase plays a central role in ceramide induced apoptosis in cultured hippocampal neurons. J Biol Chem 277: 1957-1961, 2002.

9. Yamamoto M, Hioki T, Nakajima-Iijima $S$ and Uchino $S$ : DAP kinase activity is critical for C (2)-ceramide-induced apoptosis in PC12 cells. Eur J Biochem 269: 139-147, 2002.

10. Wang WJ, Kuo JC, Yao CC and Chen RH: DAP kinase induces apoptosis by suppressing integrin activity and disrupting matrix survival signals. J Cell Biol 159: 169-179, 2002.

11. Jin Y, Blue EK, Dixon S, Hou L, Wysolmerski RB and Gallagher PJ: Identification of a new form of death-associated protein kinase that promotes cell survival. J Biol Chem 276: 39667-39678, 2001.

12. Jin Y and Gallagher PJ: Antisense depletion of death-associated protein kinase promotes apoptosis. J Biol Chem 278: 51587-51593, 2003

13. Bai T, Tanaka T, Yukawa K and Umesaki N: Reduced expression of death-associated protein kinase in human uterine and ovarian carcinoma cells. Oncol Rep 11: 661-665, 2003.
14. Li L, Tanaka T, Yukawa K, Akira S and Umesaki N: Irinotecanindiced ovarian follicular apoptosis is atteuated by deleting the kinase domain of death-associated protein kinase. Int J Oncol 34: 905-914, 2009.

15. Bai T, Tanaka T, Yukawa K and Umesaki N: A novel mechanism for acquired cisplatin-resistance: suppressed translation of death-associated protein kinase mRNA is insensitive to 5-aza-2'-deoxycitidine and trichostatin in cisplatin-resistant cervical squamous cancer cells. Int J Oncol 28: 497-508, 2006.

16. Ishiwata I, Ishiwata C, Soma M, Arai J and 0shikawa H: Establishment of human endometrial adenocarcinoma cell line containing estradiol-17 beta and progesteron receptors. Gynecol Oncol 17: 281-290, 1984.

17. Bai T, Tanaka T and Yukawa K: Targeted knockdown of deathassociated protein kinase expression induces TRAIL-mediated apoptosis in human endometrial adenocarcinoma cells. Int J Oncol 37: 203-210, 2010.

18. Tanaka T, Bai T and Yukawa K: Death-associated protein kinase is essential for the survival of various types of uterine cancer cells. Int J Oncol 37: 1017-1022, 2010.

19. Pei XH, Bai F, Tsutsui T, Kiyokawa H and Xiong Y: Genetic evidence for functional dependency of p18Ink4c on Cdk4. Mol Cell Biol 24: 6653-6664, 2004.

20. Morishita A, Masaki T, Yoshiji H, Nakai S, Ogi T, Miyauchi Y, Yoshida S, Funaki T, Uchida N, Kita Y, Funakoshi F, Usuki H, Okada S, Izushi K, Watanabe S, Kurokohchi K and Kuriyama S: Reduced expression of cell cycle regulator p18(INK4C) in human hepatocellular carcinoma. Hepatology 40: 677-686, 2004.

21. Van Veelen W, Klompmaker R, Gloerich M, van Gasteren CJ, Kalkhoven E, Berger R, Lips CJ, Medema RH, Hoeppener JW and Acton DS: P18 is a tumor suppressor gene involved in human medullary thyroid carcinoma and pheochromocytoma development. Int J Cancer 124: 339-345, 2009. 